\title{
PITIOSE CUTÂNEA CANINA - RELATO DE CASO
}

\author{
CANINE CUTANEOUS PYTHIOSIS - CASE REPORT
}

\section{M. TORRES ${ }^{1}$, A. K. F. P. DANTAS ${ }^{1}$, J. K. C. SILVA ${ }^{2}$, K. N. ARAÚJO ${ }^{1}$, F. GARINO JUNIOR ${ }^{2}$, R. S. MENDES ${ }^{2 *}$}

\begin{abstract}
RESUMO
A pitiose é uma enfermidade granulomatosa crônica, principalmente do tecido subcutâneo, causada pelo Oomiceto Pythium insidiosum que acomete humanos e animais. A espécie canina é a segunda dentre as espécies mais acometidas, sendo a manifestação clínica cutânea a forma menos comum. O sucesso da terapia é determinado pelo diagnóstico precoce da doença. A composição da parede celular torna os tradicionais fármacos antifúngicos ineficientes. Atualmente a imunoterapia se tornou uma alternativa terapêutica em potencial, entretanto, a excisão cirúrgica ainda é o principal meio de controle. Descreve-se um caso de pitiose em um cão da raça Pastor Alemão oriundo de área rural, enfocando características clínicas, aspectos histopatológicos e conduta terapêutica, onde pôde-se ressaltar que a pitiose cutânea trata-se de um importante diagnóstico diferencial dentre as dermatopatias piogranulomatosas que acomete cães, principalmente naqueles oriundos de zona rural onde possuem acesso a açudes ou áreas alagadas. De modo geral, o conhecimento do ciclo epidemiológico do $P$. insidiosum aliados a exames complementares como a histopatologia e imunohistoquímica, são ferramentas imprescindíveis em sua definição diagnóstica.
\end{abstract}

PALAVRAS-CHAVE: Cães. Cutâneo. Granulomatosa. Pythium insiduosum.

\section{SUMMARY}

Pythiosis is a chronic granulomatous disease, especially in the subcutaneous tissue caused by the oomycete Pythium insidiosum that affects humans and animals. The canine species is the second among the most affected species, and the clinical cutaneous is the least common form. The success of therapy is determined by early diagnosis. The composition of the cell wall becomes ineffective traditional antifungal drugs. Currently immunotherapy has become a potential therapeutic alternative, however, surgical excision remains the primary mean of control. We describe a case of pythiosis in a German Shepherd dog come from rural area, focusing on clinical and pathologic features, as well as therapeutic management, where it might be noted that cutaneous pythiosis is an important differential diagnosis among pyogranulomatous skin diseases affecting dogs, especially those coming from rural areas where there might be free access to ponds or wetlands. In general, knowledge of the epidemiological cycle of $P$. insidiosum combined with tests such as histopathology and immmunohistochemistry, are essential tools for its diagnosis.

KEY-WORDS: Cutaneous. Dogs. Granulomatous. Pythium insiduosum.

\footnotetext{
${ }^{1}$ Universidade Federal de Campina Grande - Centro Médico Veterinário Dr. Leonardo Torres

${ }^{2}$ Universidade Federal de Campina Grande - rodrigo.souza.mendes@ gmail.com
} 


\section{INTRODUÇÃO}

A pitiose é uma doença granulomatosa que atinge equinos, caninos, bovinos, felinos e humanos, ocorrente em áreas tropicais, subtropicais ou mesmo temperadas (MEIRELES et al., 1993; MENDOZA et al., 1993), causada pelo Oomiceto Pythium insidiosum (DE COCK et al., 1987). A espécie equina é a mais afetada, principalmente nas formas cutânea e subcutânea, seguida da canina (MENDOZA et al., 1996).

Não há predisposição por sexo, idade ou raça, obstante considera-se que caninos de maior porte são mais susceptíveis. A fonte de infecção são os zoósporos móveis aquáticos,inexistindo relatos de transmissão antropo ou zooantropozoonótica (MENDOZA et al., 1996). As condições ambientais são fundamentais para o desenvolvimento do organismo no meio ambiente. Para a produção de zoósporos são necessárias temperaturas entre 30 e $40^{\circ} \mathrm{C}$ e o acúmulo de água tépida ou quente em banhados e lagoas (MILLER \& CAMPBELL et al., 1982).

Os caninos podem apresentar a forma cutânea e gastrointestinal, sendo esta última a mais comum, caracterizada por distúrbios digestivos como êmese, anorexia crônica, emaciação, diarréia, por vezes sanguinolenta, e presença de massas nodulares, quando submetidos à palpação abdominal (FISCHER et al., 1994). A forma cutânea é caracterizada por lesões crônicas, ulceradas, nodulares, com várias extensões de drenagem, podendo acometer qualquer parte do corpo (DYKSTRA, 1999). À histopatologia, apresentam-se como dermatite piogranulomatosa ulcerativa, contendo áreas de necrose infiltrada por neutrófilos e macrófagos e granulomas eosinofílicos (FOIL et al., 1984; HOWERTH et al., 1989).

Tradicionalmente, o diagnóstico da pitiose baseava-se nas características clínicas, histopatológicas, no isolamento e na identificação do agente através de suas características culturais, morfológicas e reprodutivas. A identificação precoce da doença, no entanto, torna-se difícil por meio desses métodos. O diagnóstico diferencial deve incluir habronemose, neoplasia, quadros clínicos com tecido de granulação exuberante e os granulomas fúngicos ou bacterianos (CHAFFIN et al., 1992). Atualmente, técnicas de imunohistoquímica e sorológicas auxiliam e dão embasamento a um diagnóstico precoce e correto (MENDOZA et al., 1996).

$\mathrm{O}$ tratamento de infecções pelo $P$. insidiosum em animais e humanos é complicado devido às características do agente, sobretudo de sua composição de parede celular, uma vez que os fungos verdadeiros possuem quitina em sua parede, enquanto o Pythium insidiosum contém celulose, $\beta$-glucanos e não possuem ergosterol, que é o componente alvo de ação da maioria das drogas, tornando os fármacos antifúngicos tradicionais ineficientes contra o $P$. insidiosum (SATHAPATAYAVONGS et al., 1989; FOIL, 1996). O sucesso das outras formas de tratamento é variável, sendo influenciado pelo tamanho e duração da lesão, idade e estado nutricional do animal (MILLER, 1981). Em geral, o tratamento cirúrgico apresenta bons resultados apenas em lesões pequenas e superficiais, nas quais seja possível a retirada de toda área afetada (LEAL et al., 2001). Uma alternativa para o tratamento da pitiose equina é o protocolo imunobiológico (imunoterápico) a partir de culturas do próprio agente (hifas sonicadas). O índice de eficiência obtido na imunoterapia varia de $53 \%$ a $75 \%$ quando associado à cirurgia (MILLER, 1981; MILLER \& CAMPBELL et al., 1982). Portanto, descreve-se aqui caso de pitiose cutânea em um cão da raça Pastor Alemão originário de zona rural, enfocando características clínicas, aspectos histopatológicos e conduta terapêutica.

Um cão da raça Pastor Alemão, macho com um ano de idade foi atendido com queixa principal de lesões pruriginosas na cauda, de tal intensidade ao ponto de desenvolver automutilação, há cerca de quatro meses. Foi reportado que o animal era oriundo de uma propriedade rural, com regime de criação semidomiciliar e periodicamente tinha acesso a um açude com vegetação aquática não bem caracterizada.

Ao exame físico, o animal apresentava-se em bom estado geral, parâmetros fisiológicos (temperatura retal e frequências cardiorrespiratória) nos limites de referência para a espécie (FEITOSA, 2008), mucosas visíveis normais e linfonodos poplíteos aumentados. $\mathrm{Na}$ cauda, foi observada alopecia que se estendia da base da cauda até aproximadamente a $8^{\circ}$ vértebra coccígea, com lesões cutâneas nodulares ulceradas e exsudativas de bordos circulares e irregulares com diâmetros que variaram entre $0,5 \mathrm{~cm}$ a $3,5 \mathrm{~cm}$ de diâmetro (exsudato serosaguinolento e mucopurulento), tumefação intensa e focos de necrose (Figura 1).

O hemograma revelou apenas leucocitose (20.750 leuc/ $\left.\mathrm{mm}^{3}\right)$ com neutrofilia $\left(14.300 \mathrm{neu} / \mathrm{mm}^{3}\right)$ e eosinofilia $\left(1.452 \mathrm{eos} / \mathrm{mm}^{3}\right)$, relativo aos parâmetros sanguíneos para a espécie canina (GARCIANAVARRO \& PACHALLY, 1994). A citologia das lesões corando-se pelo método de Panótico revelou a presença de neutrófilos segmentados e eosinófilos. Alguns neutrófilos encontravam-se degenerados, havia presença de hemácias e de alguns macrófagos em pleno processo de fagocitose com restos celulares. $\mathrm{Na}$ histolopatologia cutânea foi evidenciado pela coloração de hematoxilina e eosina, intenso infiltrado inflamatório polimorfonuclear representado por neutrófilos íntegros e degenerados, plasmócitos e histiócitos permeando a derme superficial e profunda, alcançando o subcutâneo, coexistindo piogranulomas multifocais, que era compatível com dermatite piogranulomatosa. Havia áreas de necrose com infiltrado de neutrófilos e macrófagos e granulomas eosinofílicos. A coloração pelo prata (técnica de Grocott) e a técnica de imunohistoquímica revelaram, além das características anteriores, hifas bem delimitadas, bem como fragmentos fúngicos imunomarcados com coloração marrom amarelado compatíveis com as estruturas de $P$. insidiosum (Figura 2).

Antes mesmo do resultado histopatológico, ainda sob suspeita clínica inicial de pitiose cutânea, foi prescrito itraconazol $(5 \mathrm{mg} / \mathrm{kg} / \mathrm{BID} / \mathrm{VO})$, cefalexina $(30 \mathrm{mg} / \mathrm{kg} / \mathrm{BID} / \mathrm{VO})$ e meloxicam $(0,1 \mathrm{mg} / \mathrm{kg} / \mathrm{SID} / \mathrm{VO})$ até o estabelecimento diagnóstico (aproximadamente 10 dias),entretanto, não foi observada resposta 
satisfatória. Logo, mediante a definição histopatológica do quadro de pitiose, foi proposta a excisão cirúrgica, sendo o animal submetido a uma caudectomia total. Além da terapia pós-operatória antimicrobiana, antiinflamatória e analgésica, a conduta terapêutica antifúngica foi mantida após procedimento durante 60 dias, sendo o animal reavaliado clinicamente e por histopatologia aos 30 e 60 dias, onde não apresentava mais sinais de infecção por $P$. insidiosum nas proximidades do sítio cirúrgico.

A pitiose canina é uma enfermidade com distribuição mundial, de caráter crônico/progressivo, potencialmente fatal na maioria dos casos e está diretamente relacionada à exposição do cão ao ciclo de vida do $P$. insidiosum, onde os zoósporos livres encistam e emitem o tubo germinativo, dando origem a um novo micélio e, assim, completando o seu ciclo no animal (MILLER, 1983).

Os aspectos ambientais parcialmente descritos neste relato que incluíam o cão no ciclo de colonização e desenvolvimento do $P$. insidiosum se enquadram nas condições descritas por outros autores, onde os cães afetados são, normalmente, oriundos de regiões rurais ou tiveram eventual acesso a lugares alagados (FOIL el al., 1984). A grande maioria dos casos de pitiose é observada durante ou após a estação chuvosa. Baseado em dados clínico-epidemiológicos, acredita-se na existência de um período de incubação de várias semanas (LEAL et al., 2001).

Cães infectados por $P$. insidiosum, na maioria das ocasiões, manifestam lesões gastrointestinais ou cutâneas, sendo a forma gastrointestinal a mais comum e pouco frequente a forma cutânea isoladamente (MILLER, 1983; SMITH et al., 1989; FISCHER et al., 1994). É rara a ocorrência associada das apresentações clínicas em um mesmo animal (GROOTERS et al., 2003). Entretanto, em dois casos descritos no Brasil, as duas manifestações clínicas em um mesmo cão foram relatadas (NONNEMACHER et al., 2009; RECH el al., 2004.

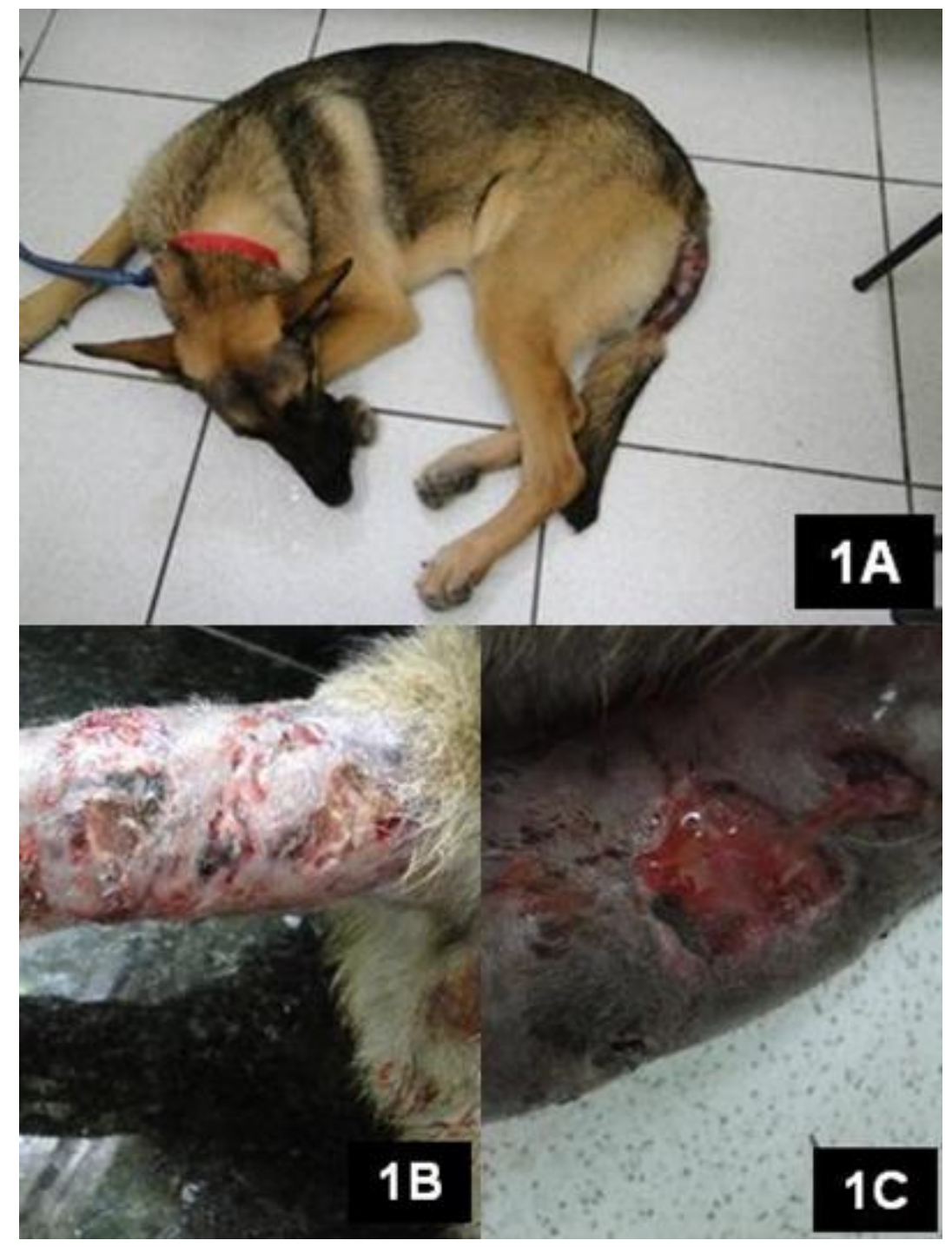

Figura 1: 1A - Cão da Raça Pastor Alemão apresentando lesão em cauda; 1B - Lesões com inflamação e ulceração cutânea com necrose multifocal (superfície direita); 1C - Lesões ulcerativas com presença de exsudato serosanguinolenta e/ muco-sanguinolenta; superfície cutânea com aspecto friável (superfície esquerda). 


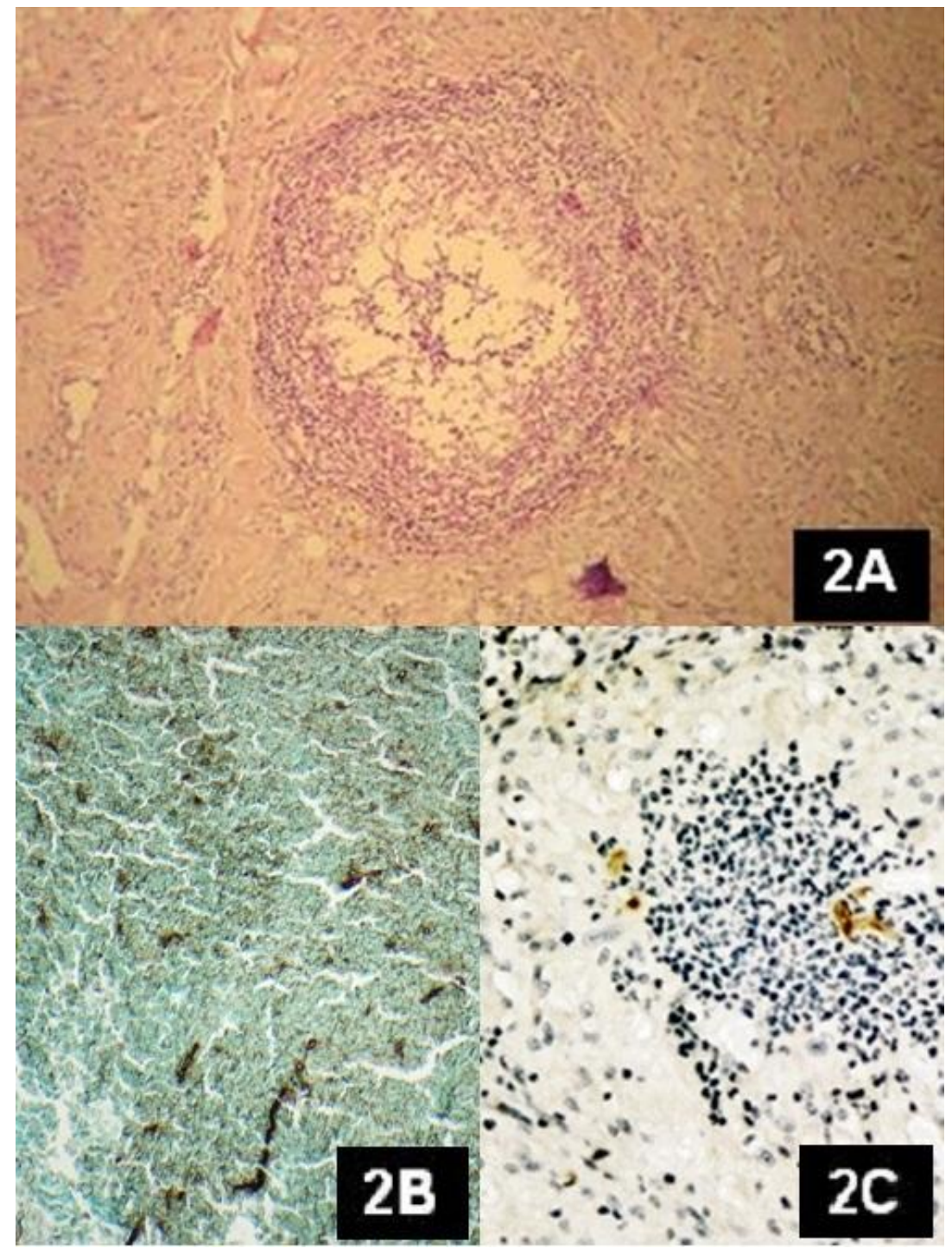

Figura 2 - Fragmento de pele de cão com Pitiose cutânea: 2A - Padrão granulomatoso, Hematoxilina e eosina (100x); 2B - Hifas evidenciadas pela coloração prata pela técnica de Grocott (400x); 2C - Presença de estruturas de coloração marrom amarelado (imunomarcados), indicando hifas e fragmentos fúngicos de $P$. insidiosum. Técnica de estreptoavidina-biotina-peroxidase (400x).

O primeiro caso de pitiose canina relatado no Brasil data de 1997, no Estado de São Paulo envolvendo fêmea com lesões cutâneas no membro pélvico esquerdo (LARSSON et al., 1997).

Os casos de pitiose cutânea geralmente acometem cães de grande porte, de um a três anos de idade (FOIL et al., 1984; DYKSTRA et al., 1999). As lesões em geral são variavelmente pruriginosas (DYKSTRA et al., 1999) e progridem rapidamente mesmo quando sob tratamento com fármacos dotados de ação antifúngica, antibiótica ou excisão cirúrgica (FOIL et al., 1984). O quadro prurítico e de automutilação observado neste caso é uma manifestação rara segundo Foil et al. (1984), podendo essa condição estar relacionada ao quadro de piodermite profunda secundária instalado, conduzindo ao estímulo periférico do prurido (GNIRS E PRÉLAUD, 2005). Os achados clínicos-patológicos do cão objeto deste relato foram similares àqueles descritos por outros autores, onde as lesões frequentemente têm evolução de um a três meses e consistem de nódulos, únicos ou múltiplos, ulcerados, de um a oito cm de diâmetro (DYKSTRA et al., 1999), ou ainda em massas ulceradas de até $30 \mathrm{~cm}$ de extensão, que envolvem tanto a pele como o panículo (FOIL et al., 1984; HOWERTH et al., 1989). Independentemente do tamanho, as lesões contêm tratos fistulosos que drenam exsudato serosanguinolento ou purulento (BENTINCK-SMITH et al., 1989; DYKSTRA et al., 1999; HENSEL et al., 2003). Em casos mais avançados, como o deste relato, a massa pode envolver, além da derme, a hipoderme. Parte desse envolvimento se deve à proliferação de tecido de granulação juntamente com áreas de necrose (BENTINCK-SMITH et al., 1989). $\mathrm{Na}$ histopatologia, o padrão de inflamação mais comum pela coloração de $\mathrm{HE}$, é de dermatite ulcerativa e piogranulomatosa (RIVIERRE et al., 2005), composta por áreas de inflamação e necrose da derme, com presença de neutrófilos e eosinófilos (HENSEL et al., 2003), às vezes combinadas a granulomas conspícuos formados unicamente por macrófagos epitelióides e células gigantes 
multinucleadas ou repletos de detritos celulares eosinofílicos (FOIL et al., 1984; HOWERTH et al., 1989). No que se refere ao tratamento, a terapia proposta neste caso foi a de excisão cirúrgica associada à terapia antifúngica a base de Itraconazol. A remoção cirúrgica das lesões continua sendo o procedimento de eleição no controle da pitiose cutânea, entretanto, bons resultados são observados apenas em lesões pequenas e superficiais, nas quais é possível a remoção de toda área afetada (LEAL et al., 2001).

Estudos avaliando a eficiência de conduta associada, excisão cirúrgica e terapia antifúngica com Anfoterricina B, demonstraram sucesso de 50\% (MCMULLAN et al., 1977). Já outros relatos revelaram que o uso isolado de drogas antifúngicas apresentam resultados variáveis in vitro e in vivo (LEAL et al., 2001), sendo o Itraconazol considerado com atividade moderada e a Terbinafina como a de maior atividade inibitória do crescimento do P. insidiosum (SHENEP et al., 1998). Naquele estudo, a associação de Terbinafina e Itraconazole apresentou efeito sinérgico resultando no sucesso do tratamento de uma criança com infecção facial. A imunoterapia é uma alternativa em potencial para o tratamento da pitiose, demonstrando em equinos cura de $53 \%$ a $83,3 \%$ e de $100 \%$ em bovinos (MONTEIRO, 1999). Por outro lado, ensaios envolvendo vacina ainda autógena produzida a partir de tecido com hifas de um cão com pitiose cutâneo são desapontadores, sendo que somente $33 \%$ dos cães responderam ao tratamento (DYKSTRA et al., 1999; MENDOZA \& NEWTON, 2005).

Um dos objetivos deste relato foi o de ressaltar a pitiose cutânea como um importante diagnóstico diferencial dentre as dermatopatias piogranulomatosas que acometem cães, tais como leishmaniose, granuloma leproide, piogranuloma estéril idiopático, celulite juvenil e micoses sistêmicas que cursam com formação de granulomas, principalmente naqueles animais oriundos de zona rural, com acesso a açudes ou áreas alagadas.

\section{REFERÊNCIAS}

BENTINCK-SMITH, J.; PAHIDE, A.; MASLIN, W. R.; HAMILTON, C.; MACDONALD, R. K.; WOODY, B. J. Canine pythiosis - isolation and identification of Pythium insidiosum. Journal of Veterinary Diagnostic Investigation, v.1, n.4, p.295-298, 1989.

CHAFFIN, M. K.; SCHUMACHER, J.; HOOPER, N. Multicentric cutaneous pythiosis in a foal. Journal of the American Animal Hospital Association, v.201, n.2, p.310-312, 1992.

DE COCK, A. W. A. M.; MENDOZA, L.; PADHYE, A. A.; ALELLO, L.; KAUFMAN, L. Pythium insidiosum sp. Nov., the etiologic agent of pythiosis. Journal Clinical Microbiology, v.25, n.2, p.344-349, 1987.

DYKSTRA, M. J.; SHARP, N. J. H.; OLIVRY, T.; HILLIER, A.; MURPHY, K.M.; KAUFMAN, L.; KUNGLE, G.A; DUCHEU-HASTON C. A description of cutaneous-subcutaneous pythiosis in fifteen dogs. Medical Mycology, v. 37, n.6, p. 427-433, 1999.

FEITOSA, F. L. F. Semiologia Veterinária - A Arte do Diagnóstico. São Paulo: Roca, 2008, p. 754.

FISCHER, J. R.; PACE, L. W.; TURK, J. R.; KREENE, J. M.; MILLER, M. A.; GOSSER, H. S. Gastrointestinal pythiosis in Missouri dogs: eleven cases. Journal of Veterinary Diagnostic Investigation, v.6, n.3, p.380382, 1994.

FOIL, C. S. O.; SHORT, B. G.; FADOK, V. A.; KUNKLE, G. A. A report of subcutaneous pythiosis in five dogs and a review of the etiologic agent Pythium spp. Journal of the American Animal Hospital Association, v.20, n.5, p.959-966, 1984.

FOIL, C. S. Update on pythiosis (Oomycosis). In: THE NORTH AMERICAN VETERINARY CONFERENCE, 1996, Orlando. USA. Anais... Orlando: Bayer Animal Health, 1996. p.57-63.

GARCIA-NAVARRO, C. E. K.; PACHALY, JR. Manual de hematologia veterinária. São Paulo: Livraria Varela Ltda, 1994. p.169.

GNIRS, K; PRÉLAUD P. Cutaneous manifestations of neurological diseases: review of neuropathophysiology and diseases causing pruritus. Veterinary Dermatology, v.16, n.3, p.137-146, 2005

GROOTERS, A. M. Pythiosis, lagenidiosis, and zygomycosis in small animals. The Veterinary Clinics of North America. Small Animal Practice, v.33, n.4, p.695-720, 2003.

HENSEL, P.; GREENE, C. E.; MEDLEAU, L. Immunotherapy for treatment of multicentric cutaneous pythiosis in a dog. Journal of the American Animal Hospital Association, v.223, n.2, p.215-218, 2003.

HOWERTH, E. W.; BROWN, C. C.; CROWDER, C. Subcutaneous pythiosis in a dog. Journal of Veterinary Diagnostic Investigation, v.1, n.1, p.8183, 1989.

LARSSON, C. E.; MENG, M. C.; NAHAS, C. R.; MICHALANY, N. S.; NEWTON, J.; ROSA, P. S.; BONAVITO, D.; GAMBALE, W.; SOARES, E. S. et al. Pitiose canina - Aspectos clínicos e epidemiológicos de caso em São Paulo. In: CONGRESSO BRASILEIRO DE MEDICINA VETERINÁRIA, 25., 1997, Gramado. Anais... Porto Alegre: Sociedade de Veterinária do Rio Grande do Sul, 1997. p.155.

LEAL, A. T.; LEAL, A. B. M.; FLORES, E. F.; SANTURIO, J. M. Pitiose. Ciência Rural, v.31, n.4, p.735-743, 2001.

MCMULLAN, W. C.; JOYCE, J. R.; HANSELKA, D. V.; HEITMANN, J. M. Amphotericin B for the treatment of localized subcutaneous phycomycosis in 
the horse. Journal of the American Animal Hospital Association, v.170, n.11, p.1293-1297, 1977.

MEIRELES, M. C. A.; RIET-CORREA, F.; FISCHMAN, O.; ZAMBRANO, A. F. H.; ZAMBRANO, M. S.; RIBEIRO, G.A. Cutaneous pythiosis in horses from Brazil. Mycoses, v.36, n.3-4, p.139-142, 1993.

MENDOZA, L.; AJELLO, L.; MCGINNIS, M.R. Infections caused by the oomycetous pathogen Pythium insidiosum. Journal Mycology Medicine, v.6, n.4, p.151164, 1996.

MENDOZA, L.; HERNANDEZ, F.; AJELLO, L. Life cycle of the human and animal oomycete pathogen Pythium insidiosum. Journal Clinical Microbiology, v.31, n.11, p.2967-2973, 1993.

MENDOZA, L.; NEWTON, J.C. Immunology and immunotherapy of the infections caused by Pythium insidiosum. Medical Mycology. v.43, n.6, p.477-486, 2005.

MILLER, R. I. Investigations into the biology of three 'phycomycotic' agents pathogenic for horses in Australia. Mycopathologia, v.81, n.1, p.23-28, 1983.

MILLER, R. I. Treatment of equine phycomycosis by immunotherapy and surgery. Australian Veterinary Journal, v.57, n.8, p.377-382, 1981.

MILLER, R. I.; CAMPBELL, R. S. F. Clinical observations on equine phycomycosis. Australian Veterinary Journal, v.58, n.6, p.221-226, 1982.

MILLER, R.I.; CAMPBELL, R.S.F. Clinical observations on equine phycomycosis. Australian Veterinary Journal, v.58, n.6, p.221-226, 1982.

MONTEIRO A. B. 1999. Imunoterapia da pitiose eqüina: teste de eficácia de um imunobiológico e avaliação leucocitária em animais infectados naturalmente pelo Pythium insidiosum. Santa Maria: Universidade Federal de Santa Maria, 1999. 52p. Dissertação (Mestrado em Medicina Veterinária) Faculdade de Medicina Veterinária, 1999.
NONNEMACHER, D. V. F.; PIZONI, C.; CORRÊA, B. F.; DE OLIVEIRA, M. P.; MOTTA, M. A, PEREIRA, C. M.; SCHILD, A. L, SALLIS VIÉGAS, E. S, PEREIRA, D. I. B. Pitiose cutânea e gastrintestinal em cão. In: XVIII CONGRESSO DE INICIAÇÃO CIENTÍFICA, 2009, Pelotas, Rio Grande do Sul. 13., 2009, Pelotas. Anais... Rio Grande do Sul: XI ENPOS/ I Mostra Científica da UFPEL , [2009] (CD-ROM).

RECH, R. R.; GRAÇA, D. L.; BARROS, C. L. S. Pitiose em um cão: Relato de caso e diagnóstico diferenciais. Revista Clínica Veterinária, v.50, n.4, p.68-72, 2004.

RIVIERRE，C.; LAPRIE，C.; GUIARD-MARIGNY, O.; BERGEAUD, P.; BERTHELEMY, M.; GUILLOT, J. Pythiosis in Africa. Emerging Infectious Diseases, v.11, n.3, p.479-481, 2005.

SATHAPATAYAVONGS，B.; LEELACHAIKUL，P.; PRACHAKTAM, R.; ATICHARTAKARN, V.; SRIPHOJANART, S.; TRAIRATVORAKUL, P.; JIRASIRITAM, S.; NONTASUT, S.; EURVILAICHIT, C.; FLEGEL, T. Human pythiosis associated with Thalassemia Hemoglobinopathy Syndrome. Journal of Infectious Disease, v.159, n.2, p.274-280, 1989.

SHENEP, J. L.; ENGLISH, B. K.; KAUFMAN, L.; PEARSON, T.A.; THOMPSON, J. W.; KAUFMAN, R. A.; FRISCH, G.; RINALDI, M. G. Successful medical therapy for deeply invasive facial infection due to Pythium insidiosum in a child. Clinical Infectious Diseases, v.27, n.6, p.1388-1393, 1998.

SMITH, J. B.; PADHYE, A. A.; MASLIN, W. R.; HAMILTON, C.; McDONALD, R. K.; WOODY, B. J. Canine pythiosis-isolation and identification of Pythium insidiosum. Journal of Veterinary Diagnostic Investigation, v.1, n.4, p.295-298, 1989. 